\title{
Robust Multiresolution Coding
}

\author{
Jun Chen, Member, IEEE, Sorina Dumitrescu, Member, IEEE, Ying Zhang, and Jia Wang
}

\begin{abstract}
In multiresolution coding a source sequence is encoded into a base layer and a refinement layer. The refinement layer, constructed using a conditional codebook, is in general not decodable without the correct reception of the base layer. By relating multiresolution coding with multiple description coding, we show that it is in fact possible to construct multiresolution codes in certain ways so that the refinement layer alone can be used to reconstruct the source to achieve a nontrivial distortion. As a consequence, one can improve the robustness of the existing multiresolution coding schemes without sacrificing the efficiency. Specifically, we obtain an explicit expression of the minimum distortion achievable by the refinement layer for arbitrary finite alphabet sources with Hamming distortion measure. Experimental results show that the information-theoretic limits can be approached using a practical robust multiresolution coding scheme based on low-density generator matrix codes.
\end{abstract}

Index Terms-Low-density generator matrix, message-passing algorithm, multiple description coding, multiresolution coding, successive refinement.

\section{INTRODUCTION}

$\mathbf{M}$ ANY important applications require multicast delivery of data from a single user to multiple receivers with diverse characteristics in terms of bandwidth resources, computational capabilities, and fidelity requirements. It is desirable that the reconstruction quality at each receiver is commensurate with its own demand and capability. As a promising solution to this problem, multiresolution coding has received significant attention in recent years [1]-[8].

In multiresolution coding a source sequence is encoded into a base layer and a refinement layer; a coarse reconstruction of the source is possible based on the base layer while the two layers together can lead to better reconstruction quality. Although it is commonly assumed that the role of the refinement layer is simply to improve the reconstruction precision upon that achieved by the base layer, it is of considerable interest to know whether the refinement layer alone can be used to reconstruct the source. Unfortunately, for most existing multiresolution coding schemes, the refinement layer is constructed using a conditional codebook, thus is undecodable without the correct reception of the base layer or is essentially useless for producing any nontrivial reconstruction. By interpreting multiresolution coding as a special case of

Paper approved by Z. Xiong, the Editor for Distributed Coding and Processing of the IEEE Communications Society. Manuscript received January 12, 2010; revised April 8, 2010.

J. Chen, S. Dumitrescu, and Y. Zhang are with the Department of Electrical and Computer Engineering, McMaster University, Hamilton, ON L8S 4K1, Canada (e-mail: \{junchen, sorina\}@mail.ece.mcmaster.ca, yingzhang@grads.ece.mcmaster.ca).

J. Wang is with the Department of Electronic Engineering, Shanghai Jiao Tong University, Shanghai 200240, China (e-mail: jiawang@sjtu.edu.cn).

The material in this paper was presented in part at the IEEE International Symposium on Information Theory, Austin, Texas, USA, June 13-18, 2010.

Digital Object Identifier 10.1109/TCOMM.2010.093010.100024 multiple description coding and leveraging relevant multiple description code constructions, we shall show that it is in fact possible to design multiresolution codes in certain ways so that the refinement layer alone can be (partially) decoded to produce a nontrivial reconstruction of the source. This is certainly a desirable feature since it improves the robustness of multiresolution codes without sacrificing the efficiency.

The remainder of this paper is organized as follows. In Section II, we discuss the connection between multiresolution coding and multiple description coding. Some existing results on these two coding problems are reviewed. In Section III, we derive an explicit expression of the minimum distortion achievable by the refinement layer for arbitrary finite alphabet sources with Hamming distortion measure. A practical robust multiresolution coding scheme based on low-density generator matrix (LDGM) codes is proposed in Section IV. The effectiveness of the proposed scheme is verified in Section V. Finally, we conclude the paper in Section VI.

\section{Multiresolution Coding And Multiple DESCRIPTION CODING}

We shall first review some basic definitions and results regarding multiresolution coding and multiple description coding. It will be seen that a key step toward understanding the role of refinement layer in multiresolution coding is to interpret multiresolution coding as a special form of multiple description coding.

\section{A. Multiple Description Coding}

In the multiple description problem, a source sequence is encoded into two descriptions, which are constructed in such a way that an adequate reconstruction of the source is possible based on each description while the two descriptions together can lead to better reconstruction quality. A fundamental problem of multiple description coding is to characterize the ratedistortion region, which determines the information-theoretic limits of multiple description coding.

Consider an i.i.d. process $\{X(l)\}_{l=1}^{\infty}$ with marginal distribution $p_{X}$ on source alphabet $\mathcal{X}$. Let $d: \mathcal{X} \times \hat{\mathcal{X}} \rightarrow[0, \infty)$ be a distortion measure, where $\hat{\mathcal{X}}$ is the reconstruction alphabet. We assume that $\mathcal{X}$ and $\hat{\mathcal{X}}$ are finite sets.

Definition 1: The quintuple $\left(R_{1}, R_{2}, D_{0}, D_{1}, D_{2}\right)$ is said achievable, if for all sufficiently large $n$, there exist encoding functions

$$
f_{i}^{(n)}: \mathcal{X}^{n} \rightarrow\left\{1,2, \cdots,\left\lfloor 2^{n R_{i}}\right\rfloor\right\}, \quad i=1,2,
$$

and decoding functions

$$
\begin{aligned}
& g_{0}^{(n)}:\left\{1,2, \cdots,\left\lfloor 2^{n R_{1}}\right\rfloor\right\} \times\left\{1,2, \cdots,\left\lfloor 2^{n R_{2}}\right\rfloor\right\} \rightarrow \hat{\mathcal{X}}^{n}, \\
& g_{i}^{(n)}:\left\{1,2, \cdots,\left\lfloor 2^{n R_{i}}\right\rfloor\right\} \rightarrow \hat{\mathcal{X}}^{n}, \quad i=1,2,
\end{aligned}
$$


such that

$$
\mathbb{E}\left[\frac{1}{n} \sum_{l=1}^{n} d\left(X(l), X_{i}(l)\right)\right] \leq D_{i}, \quad i=0,1,2,
$$

where $X_{0}^{n}=g_{0}^{(n)}\left(f_{1}^{(n)}\left(X^{n}\right), f_{2}^{(n)}\left(X^{n}\right)\right)$ and $X_{i}^{n}=$ $g_{i}^{(n)}\left(f_{i}^{(n)}\left(X^{n}\right)\right), i=1,2$. The multiple description ratedistortion region, denoted by $\mathcal{R} \mathcal{D}_{\mathrm{MD}}$, is the closure of the set of all achievable quintuples $\left(R_{1}, R_{2}, D_{0}, D_{1}, D_{2}\right)$.

While a computable characterization of $\mathcal{R} \mathcal{D}_{\mathrm{MD}}$ is still unknown, several inner bounds of $\mathcal{R} \mathcal{D}_{\mathrm{MD}}$ can be found in the literature [9]-[11], among which the EGC inner bound [9] is the one that is particularly relevant to our setting. Specifically, the EGC inner bound $\mathcal{R} \mathcal{D}_{\mathrm{EGC}}$ is the convex hull of the set of quintuples $\left(R_{1}, R_{2}, D_{0}, D_{1}, D_{2}\right)$ for which there exist auxiliary random variables $X_{i}, i=0,1,2$, jointly distributed with the generic source variable $X$, such that

$$
\begin{aligned}
& R_{i} \geq I\left(X ; X_{i}\right), \quad i=1,2, \\
& R_{1}+R_{2} \geq I\left(X ; X_{0}, X_{1}, X_{2}\right)+I\left(X_{1} ; X_{2}\right), \\
& D_{i} \geq \mathbb{E}\left[d\left(X, X_{i}\right)\right], \quad i=0,1,2 .
\end{aligned}
$$

The coding scheme associated with the EGC inner bound can be roughly understood as follows. Generate codebook 1 and codebook 2 using marginal distributions $p_{X_{1}}$ and $p_{X_{2}}$, respectively. For each pair of codewords, one from codebook 1 and the other from codebook 2 , generate a codebook using the conditional distribution $p_{X_{0} \mid X_{1} X_{2}}$; such a codebook will be referred to as a conditional codebook. The source $X$ is encoded into two descriptions, where description 1 contains an index specifying a codeword $X_{1}$ in codebook 1 and a portion of index specifying a codeword $X_{0}$ in the conditional codebook while description 2 contains an index specifying a codeword $X_{2}$ in codebook 2 and the remaining portion of index for $X_{0}$. Here the conditional codebook itself is specified by $X_{1}$ and $X_{2}$ (or equivalently, the indices of $X_{1}$ and $X_{2}$ ). Given a single description, say, description $i$, one can decode $X_{i}$ and use it as the reconstruction of $X$. If both descriptions are received, then one can decode $X_{0}$ and use it as the reconstruction. Note that given a single description, it is in general impossible to (even partially) decode $X_{0}$ since the available information is not enough to specify the conditional codebook from which $X_{0}$ is picked; moreover, if such a description only contains a partial index for $X_{0}$, then the position of $X_{0}$ in the conditional codebook is also ambiguous.

\section{B. Multiresolution Coding}

It is instructive to view multiresolution coding as a special form of multiple description coding in which the distortion constraint on the second description (i.e., $D_{2}$ ) is not imposed. In this scenario it is common to refer to the first description as the base layer and the second description as the refinement layer.

Definition 2: The multiresolution coding rate-distortion region $\mathcal{R} \mathcal{D}_{\text {MR }}$ is given by

$$
\mathcal{R D}_{\mathrm{MR}}=\left\{\left(R_{1}, R_{2}, D_{0}, D_{1}\right):\left(R_{1}, R_{2}, D_{0}, D_{1}, \infty\right) \in \mathcal{R} \mathcal{D}_{\mathrm{MD}}\right\} .
$$

As shown in [12], $\mathcal{R} \mathcal{D}_{\mathrm{MR}}$ is equal to the set of quadruples $\left(R_{1}, R_{2}, D_{0}, D_{1}\right)$ for which there exist auxiliary random vari- ables $X_{0}$ and $X_{1}$, jointly distributed with the generic source variable $X$, such that

$$
\begin{aligned}
& R_{1} \geq I\left(X ; X_{1}\right), \\
& R_{1}+R_{2} \geq I\left(X ; X_{0}, X_{1}\right), \\
& D_{i} \geq \mathbb{E}\left[d\left(X, X_{i}\right)\right], \quad i=0,1 .
\end{aligned}
$$

It is easy to see that $\mathcal{R} \mathcal{D}_{\mathrm{MR}}$ is equivalent to $\mathcal{R} \mathcal{D}_{\mathrm{EGC}}$ with $X_{2}$ set to be a constant. Such a connection is well understood. In fact, most existing multiresolution code constructions are based on this interpretation of $\mathcal{R} \mathcal{D}_{\mathrm{MR}}$. However, this interpretation has the following implicit consequence on the resulting constructions, that is, the refinement layer alone is in general useless for reconstructing the source. Indeed, in the aforedescribed EGC scheme, if one sets $X_{2}$ to be a constant, then the second description alone is in general not (even partially) decodable since it only contains a portion of index specifying a codeword $X_{0}$ in the conditional codebook. It will be seen that there is an alternative way to design the refinement layer based on a deeper connection between $\mathcal{R} \mathcal{D}_{\text {MR }}$ and $\mathcal{R} \mathcal{D}_{\text {EGC. }}$

\section{Connection}

In the most general formulation, if one simply imposes the requirement that the refinement layer alone can be used to produce a nontrivial reconstruction of the source, then multiresolution coding becomes equivalent to multiple description coding. In practice, multiresolution coding often has a more restricted meaning: loosely speaking, the base layer and the refinement layer should be constructed in a greedy manner to achieve the minimum distortion at each reconstruction step. This is the case where multiresolution coding is most interesting. Indeed, such a greedy property can even be viewed as the essential feature of multiresolution coding. We shall show that in this natural setting it is possible to determine the minimum distortion achievable by the refinement layer of a multiresolution code.

Let $R(D)$ denote the rate-distortion function, i.e., $R(D)=$ $\min _{p_{\hat{X} \mid X}} I(X ; \hat{X})$, where the minimization is over $p_{\hat{X} \mid X}$ subject to the constraint $\mathbb{E}[d(X, \hat{X})] \leq D$. Define

$$
R\left(R_{1}, D_{0}, D_{1}\right)=\min \left\{R_{1}+R_{2}:\left(R_{1}, R_{2}, D_{0}, D_{1}\right) \in \mathcal{R} \mathcal{D}_{\mathrm{MR}}\right\} .
$$

It can be shown [12] that

$$
R\left(R_{1}, D_{0}, D_{1}\right)=\max \left\{R_{1}, \min _{p_{X_{0} X_{1} \mid X}} I\left(X ; X_{0}, X_{1}\right)\right\},
$$

where the minimization is over $p_{X_{0} X_{1} \mid X}$ subject to the constraints $\mathbb{E}\left[d\left(X, X_{0}\right)\right] \leq D_{0}, I\left(X ; X_{1}\right) \leq R_{1}$, and $\mathbb{E}\left[d\left(X, X_{1}\right)\right] \leq D_{1}$. Define

$$
D_{2}^{*}\left(D_{0}, D_{1}\right)=\min _{\substack{R_{1}=R\left(D_{1}\right) \\ R_{1}+R_{2}=R\left(R_{1}, D_{0}, D_{1}\right) \\\left(R_{1}, R_{2}, D_{0}, D_{1}, D_{2}\right) \in \mathcal{R} \mathcal{D}_{\mathrm{MD}}}} D_{2} .
$$

Note that $D_{2}^{*}\left(D_{0}, D_{1}\right)$ can be interpreted as the minimum distortion achievable by the refinement layer in the case where $R_{1}=R\left(D_{1}\right)$ and $R_{1}+R_{2}=R\left(R\left(D_{1}\right), D_{0}, D_{1}\right)$. The following result is a simple consequence of [13, Lemma 3].

Theorem 1: Let $\mathcal{Q}$ denote the convex hull of the set of quintuples $\left(R_{1}, R_{2}, D_{0}, D_{1}, D_{2}\right)$ for which there exist auxiliary 
random variables $X_{i}, i=0,1,2$, jointly distributed with the generic source variable $X$, such that

$$
\begin{aligned}
& I\left(X_{1} ; X_{2}\right)=0, \\
& R_{i} \geq I\left(X ; X_{i}\right), \quad i=1,2, \\
& R_{1}+R_{2} \geq I\left(X ; X_{0}, X_{1}, X_{2}\right), \\
& D_{i} \geq \mathbb{E}\left[d\left(X, X_{i}\right)\right], \quad i=0,1,2 .
\end{aligned}
$$

We have

$$
D_{2}^{*}\left(D_{1}, D_{0}\right)=\min _{\substack{R_{1}=R\left(D_{1}\right) \\ R_{1}+R_{2}=R\left(R_{1}, D_{0}, D_{1}\right) \\\left(R_{1}, R_{2}, D_{0}, D_{1}, D_{2}\right) \in \mathcal{Q}}} D_{2}
$$

Remark: This result can also be proved by invoking [14, Theorem 1] if $R\left(R_{1}, D_{1}, D_{0}\right)=R\left(D_{0}\right)$.

Note that one can obtain $\mathcal{Q}$ from $\mathcal{R} \mathcal{D}_{\mathrm{EGC}}$ by imposing an additional constraint $I\left(X_{1} ; X_{2}\right)=0$ (i.e., $X_{1}$ and $X_{2}$ are independent). This reveals a new perspective on multiresolution coding. Roughly speaking, to obtain a multiresolution coding scheme from an EGC scheme, one just needs to let $X_{2}$ be independent of $X_{1}$, instead of setting $X_{2}$ to be a constant. In this way, the refinement layer alone is still useful since one can decode $X_{2}$ and use it as the reconstruction of the source.

In principle it is possible to compute $D_{2}^{*}\left(D_{0}, D_{1}\right)$ by solving the minimization problem in (1) via numerical methods. In the next section we shall derive an explicit expression of $D_{2}^{*}\left(D_{0}, D_{1}\right)$ for arbitrary finite alphabet sources with Hamming distortion measure.

\section{Finite Alphabet Source With Hamming Distortion MEASURE}

Let $\mathcal{X}=\hat{\mathcal{X}}=\{0,1, \cdots, m\}$ for some positive integer $m$. Let $d=d_{H}: \mathcal{X} \times \hat{\mathcal{X}} \rightarrow\{0,1\}$ be the Hamming distortion measure, i.e, $d_{H}(x, \hat{x})=0$ if $x=\hat{x}$ and $d_{H}(x, \hat{x})=1$ if $x \neq \hat{x}$. Note that $\mathbb{E}\left[d_{H}(X, \hat{X})\right]=\mathbb{P}(X \neq \hat{X})$. Without loss of generality, we shall assume $p_{X}(0) \geq p_{X}(1) \geq \cdots \geq$ $p_{X}(m)>0$.

Let $R(D)$ and $D(R)$ denote the rate-distortion function and the distortion-rate function, respectively, of source $X$ with Hamming distortion measure. The following facts are well known [15]-[17].

F1) $\left.D(R)\right|_{R=0}=1-\max _{x \in \mathcal{X}} p_{X}(x)=1-p_{X}(0)$.

F2) $R(D)$ is a strictly convex function of $D$ for $D \in$ $\left[0,\left.D(R)\right|_{R=0}\right]$.

F3) For $0 \leq D_{0} \leq D_{1}<\left.D(R)\right|_{R=0}$, we have

$$
\begin{aligned}
& I\left(X ; X_{1}\right)=R\left(D_{1}\right), \\
& I\left(X ; X_{0}, X_{1}\right)=R\left(D_{0}\right), \\
& \mathbb{P}\left(X \neq X_{i}\right)=D_{i}, \quad i=0,1,
\end{aligned}
$$

if and only if $X-X_{0}-X_{1}$ form a Markov chain, and $p_{X X_{0} X_{1}}$ is specified by

$$
p_{X_{i}}(x)=\frac{\left(p_{X}(x)-\lambda_{i}\right)^{+}}{\sum_{x^{\prime} \in \mathcal{X}}\left(p_{X}\left(x^{\prime}\right)-\lambda_{i}\right)^{+}}, \quad x \in \mathcal{X}, i=0,1,
$$

$$
\begin{aligned}
& p_{X \mid X_{0}}\left(x \mid x_{0}\right) \\
& = \begin{cases}1-D_{0}, & x=x_{0} \in \mathcal{X}_{0}^{+} \\
\lambda_{0}, & x \neq x_{0}, x \in \mathcal{X}_{0}^{+}, x_{0} \in \mathcal{X}_{0}^{+}, \\
p_{X}(x) & x \notin \mathcal{X}_{0}^{+}, x_{0} \in \mathcal{X}_{0}^{+}\end{cases} \\
& p_{X_{0} \mid X_{1}}\left(x_{0} \mid x_{1}\right) \\
& =\left\{\begin{array}{ll}
\frac{1-D_{1}-\lambda_{0}}{1-D_{0}-\lambda_{0}}, & x_{0}=x_{1} \in \mathcal{X}_{1}^{+} \\
\frac{\lambda_{1}-\lambda_{0}}{1-D_{0}-\lambda_{0}}, & x_{0} \neq x_{1}, x_{0} \in \mathcal{X}_{1}^{+}, x_{1} \in \mathcal{X}_{1}^{+} \\
\frac{p_{X}\left(x_{0}\right)-\lambda_{0}}{1-D_{0}-\lambda_{0}} & x_{0} \in \mathcal{X}_{0}^{+}, x_{0} \notin \mathcal{X}_{1}^{+}, x_{1} \in \mathcal{X}_{1}^{+}
\end{array},\right.
\end{aligned}
$$

where $\lambda_{0} \in\left[0, \lambda_{1}\right]$ and $\lambda_{1} \in\left[0, p_{X}(1)\right)$ are uniquely determined by

$$
\sum_{x_{i} \in \mathcal{X}_{i}^{+}} p_{X_{i}}\left(x_{i}\right) p_{X \mid X_{i}}\left(x \mid x_{i}\right)=p_{X}(x), \quad x \in \mathcal{X}, i=0,1,
$$

and $\mathcal{X}_{i}^{+}=\left\{x \in \mathcal{X}: p_{X}(x)-\lambda_{i}>0\right\}, i=0,1$.

F4) $R\left(R\left(D_{1}\right), D_{0}, D_{1}\right)=R\left(D_{0}\right)$ for $0 \leq D_{0} \leq D_{1} \leq$ $\left.D(R)\right|_{R=0}$.

The following theorem is the main result of this section.

Theorem 2: $D_{2}^{*}\left(D_{1}, D_{0}\right)=\left.D(R)\right|_{R=0}+D_{0}-D_{1}$ for $0 \leq$ $D_{0} \leq D_{1} \leq\left. D(R)\right|_{R=0}$.

Remark: For the binary uniform source, Theorem 2 reduces to [13, Theorem 5]. Moreover, it is interesting to note that Theorem 2 also holds for the quadratic Gaussian case [18].

We shall prove Theorem 2 by establishing a series of lemmas. It is clear that Theorem 2 is true when $D_{1}=$ $\left.D(R)\right|_{R=0}$. Therefore, we shall only consider the case $0 \leq$ $D_{0} \leq D_{1}<\left.D(R)\right|_{R=0}$. For $0 \leq D_{0} \leq D_{1}<\left.D(R)\right|_{R=0}$, let $p_{X X_{0} X_{1}}$ be the probability distribution specified by (2)(4) and the Markov chain constraint $X-X_{0}-X_{1}$; define $\tilde{D}_{2}\left(D_{0}, D_{1}\right)=\min _{p_{X_{2} \mid X X_{0} X_{1}}} \mathbb{P}\left(X \neq X_{2}\right)$, where the minimization is over $p_{X_{2} \mid X X_{0} X_{1}}$ subject to the constraints $I\left(X ; X_{1}, X_{2} \mid X_{0}\right)=0$ (i.e., $X-X_{0}-\left(X_{1}, X_{2}\right)$ form a Markov chain) and $I\left(X_{1} ; X_{2}\right)=0$ (i.e., $X_{1}$ and $X_{2}$ are independent).

Lemma 1: $D_{2}^{*}\left(D_{0}, D_{1}\right)=\tilde{D}_{2}\left(D_{0}, D_{1}\right)$ for $0 \leq D_{0} \leq$ $D_{1}<\left.D(R)\right|_{R=0}$.

Proof: For any $X_{2}$ such that $I\left(X ; X_{1}, X_{2} \mid X_{0}\right)=0$ and $I\left(X_{1} ; X_{2}\right)=0$, let

$$
\begin{aligned}
& R_{1}=I\left(X ; X_{1}\right), \\
& R_{2}=I\left(X, X_{1} ; X_{2}\right)+I\left(X ; X_{0} \mid X_{1}, X_{2}\right), \\
& D_{i}=\mathbb{P}\left(X \neq X_{i}\right), \quad i=0,1,2 .
\end{aligned}
$$

Note that $R_{2} \geq I\left(X ; X_{2}\right)$ and

$$
\begin{aligned}
R_{1}+R_{2}= & I\left(X ; X_{1}\right)+I\left(X, X_{1} ; X_{2}\right)+I\left(X ; X_{0} \mid X_{1}, X_{2}\right) \\
= & I\left(X ; X_{1}\right)+I\left(X_{1} ; X_{2}\right)+I\left(X ; X_{2} \mid X_{1}\right) \\
& +I\left(X ; X_{0} \mid X_{1}, X_{2}\right) \\
= & I\left(X ; X_{1}\right)+I\left(X ; X_{2} \mid X_{1}\right)+I\left(X ; X_{0} \mid X_{1}, X_{2}\right) \\
= & I\left(X ; X_{0}, X_{1}, X_{2}\right) .
\end{aligned}
$$

Therefore, we have $\left(R_{1}, R_{2}, D_{0}, D_{1}, D_{2}\right) \in \mathcal{Q}$. Moreover, since

$$
\begin{aligned}
& R_{1}=I\left(X ; X_{1}\right)=R\left(D_{1}\right), \\
& R_{1}+R_{2}=I\left(X ; X_{0}, X_{1}, X_{2}\right)=I\left(X ; X_{0}, X_{1}\right)=R\left(D_{0}\right), \\
& D_{i}=\mathbb{P}\left(X \neq X_{i}\right), \quad i=0,1,
\end{aligned}
$$


it follows from F4) and Theorem 1 that $D_{2}^{*}\left(D_{0}, D_{1}\right) \leq \mathbb{P}(X \neq$ $\left.X_{2}\right)$, which further implies that $D_{2}^{*}\left(D_{0}, D_{1}\right) \leq \tilde{D}_{2}\left(D_{0}, D_{1}\right)$.

Now we proceed to show that $D_{2}^{*}\left(D_{0}, D_{1}\right) \geq \tilde{D}_{2}\left(D_{0}, D_{1}\right)$. In view of F4) and Theorem 1, we have $\left(R\left(D_{1}\right), R\left(D_{0}\right)-\right.$ $\left.R\left(D_{1}\right), D_{0}, D_{1}, D_{2}^{*}\left(D_{0}, D_{1}\right)\right) \in \mathcal{Q}$. By the definition of $\mathcal{Q}$, there exist $p_{X X_{0}^{(j)} X_{1}^{(j)} X_{2}^{(j)}}$ and $\mu_{j}>0, j=1,2, \cdots, r$, for some positive integer $r$ such that

$$
\begin{aligned}
& I\left(X_{1}^{(j)} ; X_{2}^{(j)}\right)=0, \quad j=1,2, \cdots, r, \\
& \sum_{j=1}^{r} \mu_{j}=1, \\
& \sum_{j=1}^{r} \mu_{j} I\left(X ; X_{1}^{(j)}\right) \leq R\left(D_{1}\right), \\
& \sum_{j=1}^{r} \mu_{j} I\left(X ; X_{0}^{(j)}, X_{1}^{(j)}, X_{2}^{(j)}\right) \leq R\left(D_{0}\right), \\
& \sum_{j=1}^{r} \mu_{j} \mathbb{P}\left(X \neq X_{i}^{(j)}\right) \leq D_{i}, \quad i=0,1, \\
& \sum_{j=1}^{r} \mu_{j} \mathbb{P}\left(X \neq X_{2}^{(j)}\right) \leq D_{2}^{*}\left(D_{0}, D_{1}\right) .
\end{aligned}
$$

It can be shown by leveraging F2) that

$$
\begin{aligned}
& I\left(X ; X_{i}^{(j)}\right)=R\left(D_{i}\right), \quad i=0,1, \quad j=1,2, \cdots, r, \\
& \mathbb{P}\left(X \neq X_{i}^{(j)}\right)=D_{i}, \quad i=0,1, \quad j=1,2, \cdots, r .
\end{aligned}
$$

By (8) and (11), we must have

$$
\begin{gathered}
I\left(X ; X_{0}^{(j)}, X_{1}^{(j)}, X_{2}^{(j)}\right)=I\left(X ; X_{0}^{(j)}, X_{1}^{(j)}\right)=I\left(X ; X_{0}^{(j)}\right), \\
j=1,2, \cdots, r,
\end{gathered}
$$

i.e., $I\left(X ; X_{1}^{(j)}, X_{2}^{(j)} \mid X_{0}^{(j)}\right)=0, j=1,2, \cdots, r$. In view of (11), (12), and (13), one can readily show by invoking F3) that $p_{X X_{0}^{(j)} X_{1}^{(j)}}=p_{X X_{0} X_{1}}, j=1,2, \cdots, r$. Therefore, it follows from (10) and the definition of $\tilde{D}_{2}\left(D_{0}, D_{1}\right)$ that

$$
D_{2}^{*}\left(D_{0}, D_{1}\right) \geq \min _{j \in\{1,2, \cdots, r\}} \mathbb{P}\left(X \neq X_{2}^{(j)}\right) \geq \tilde{D}_{2}\left(D_{0}, D_{1}\right) .
$$

The proof is complete.

It is easy to see from the definition of $\mathcal{X}_{0}^{+}$and $\mathcal{X}_{1}^{+}$that $\mathcal{X}_{i}^{+}=\left\{0,1, \cdots, m_{i}\right\}, i=0,1$, for some positive integers $m_{0}$ and $m_{1}$; moreover, we have $\mathcal{X}_{1}^{+} \subseteq \mathcal{X}_{0}^{+}$(i.e., $m_{1} \leq m_{0}$ ). Let $\mathcal{P}\left(\mathcal{X}_{0}^{+}\right)$denote the set of probability distributions defined on $\mathcal{X}_{0}^{+}$.

Lemma 2: With no loss of generality one can assume $p_{X_{2}} \in$ $\mathcal{P}\left(\mathcal{X}_{0}^{+}\right)$in the definition of $\tilde{D}_{2}\left(D_{0}, D_{1}\right)$.

Proof: See Appendix A.

Lemma 3: For any $X_{2}$ such that $p_{X_{2}} \in \mathcal{P}\left(\mathcal{X}_{0}^{+}\right)$and $I\left(X ; X_{2} \mid X_{0}\right)=0$, we have

$$
\mathbb{P}\left(X \neq X_{2}\right)=D_{0}+\left(1-D_{0}-\lambda_{0}\right) \mathbb{P}\left(X_{0} \neq X_{2}\right) .
$$

Proof: See Appendix B.

Define $\bar{D}_{2}\left(D_{0}, D_{1}\right)=\min _{p_{X_{2} \mid X_{0} X_{1}}} \mathbb{P}\left(X_{0} \neq X_{2}\right)$, where the minimization is over $p_{X_{2} \mid X_{0} X_{1}}$ subject to the constraints $p_{X_{2}} \in \mathcal{P}\left(\mathcal{X}_{0}^{+}\right)$and $I\left(X_{1} ; X_{2}\right)=0$. It is obvious that $\bar{D}_{2}\left(D_{0}, D_{1}\right)$ is unaffected if the constraint $p_{X_{2}} \in \mathcal{P}\left(\mathcal{X}_{0}^{+}\right)$is removed.
Lemma 4: $\tilde{D}_{2}\left(D_{0}, D_{1}\right)=D_{0}+\left(1-D_{0}-\lambda_{0}\right) \bar{D}_{2}\left(D_{0}, D_{1}\right)$.

Proof: In view of the fact that $D_{0} \leq\left. D(R)\right|_{R=0}=1-$ $p_{X}(0)$ (see F1)) and $\lambda_{0} \leq \lambda_{1} \leq p_{X}(1) \leq p_{X}(0)$ (see F3)), we have $1-D_{0}-\lambda_{0} \geq 0$. Therefore, this result is a direct consequence of Lemma 2 and Lemma 3.

Lemma 5: $\bar{D}_{2}\left(D_{0}, D_{1}\right)=1-p_{X_{0}}(0)-\mathbb{P}\left(X_{0} \neq X_{1}\right)$.

Proof: See Appendix C.

Combining Lemmas 1, 4, and 5, we have

$$
\begin{aligned}
& D_{2}^{*}\left(D_{0}, D_{1}\right) \\
& =D_{0}+\left(1-D_{0}-\lambda_{0}\right)\left(1-p_{X_{0}}(0)-\mathbb{P}\left(X_{0} \neq X_{1}\right)\right) \\
& =D_{0}+\left(1-D_{0}-\lambda_{0}\right)\left(1-p_{X_{0}}(0)-1+\frac{1-D_{1}-\lambda_{0}}{1-D_{0}-\lambda_{0}}\right) \\
& =1-\left(1-D_{0}\right) p_{X_{0}}(0)-\lambda_{0}\left(1-p_{X_{0}}(0)\right)+D_{0}-D_{1} \\
& =1-p_{X}(0)+D_{0}-D_{1} \\
& =\left.D(R)\right|_{R=0}+D_{0}-D_{1}
\end{aligned}
$$

where (14), (15), and (16) follow from (4), (3), and F1), respectively. This completes the proof of Theorem 2 .

\section{Practical Robust Multiresolution Coding SCHEME}

We shall present a robust multiresolution coding scheme based on LDGM codes.

It is instructive to first explain the underlying ideas using random codes and joint typicality encoding.

1) Codebook Generation: Generate two random codebooks $\mathcal{C}_{1}=\left\{x_{1, k_{1}}^{n}\right\}_{k_{1}=1}^{2^{n\left(I\left(X ; X_{1}\right)+\epsilon_{1}\right)}}$ and $\mathcal{C}_{2}=$

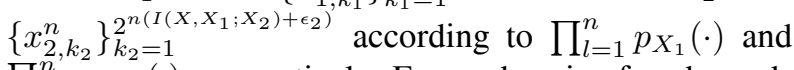
$\prod_{l=1}^{n} p_{X_{2}}(\cdot)$, respectively. For each pair of codewords $x_{1, k_{1}}^{n} \in \mathcal{C}_{1}$ and $x_{2, k_{2}}^{n} \in \mathcal{C}_{2}$, generate a random codebook

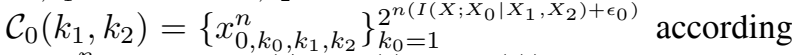
to $\prod_{l=1}^{n} p_{X_{0} \mid X_{1} X_{2}}\left(\cdot \mid x_{1, k_{1}}(l), x_{2, k_{2}}(l)\right)$.

2) Encoding: Given the source sequence $x^{n}$, first find $k_{1}^{*}$ such that $x_{1, k_{1}^{*}}^{n}$ is jointly strongly typical with $x^{n}$, then find $k_{2}^{*}$ such that $x_{2, k_{2}^{*}}^{n}$ is jointly strongly typical with $\left(x^{n}, x_{1, k_{1}^{*}}^{n}\right)$, finally find $k_{0}^{*}$ such that $x_{0, k_{0}^{*}, k_{1}^{*}, k_{2}^{*}}$ is jointly strongly typical with $\left(x^{n}, x_{1, k_{1}^{*}}^{n}, x_{2, k_{2}^{*}}^{n}\right)$. The base layer of the multiresolution code contains the index $k_{1}^{*}$ while the refinement layer contains $k_{2}^{*}$ and $k_{0}^{*}$.

3) Decoding: The decoder uses $x_{1, k_{1}^{*}}^{n}$ as the reconstruction if the base layer is received, uses $x_{2, k_{2}^{*}}^{n}$ as the reconstruction if the refinement layer is received, and uses $x_{0, k_{0}^{*}, k_{1}^{*}, k_{2}^{*}}^{n}$ as the reconstruction if both layers are received.

Notice that the encoder can be regarded as the cascade of three encoders $\mathcal{E}_{1}, \mathcal{E}_{2}$, and $\mathcal{E}_{3}$ outputting indices $k_{1}^{*}, k_{2}^{*}$, and $k_{0}^{*}$, respectively. Following the above theoretical coding system we propose a practical coding scheme, which employs a multilevel LDGM code to generate the codebook, in conjunction with a message passing algorithm, at each component encoder. Multilevel LDGM codes were introduced in [19] to generate codebooks with codewords of non-uniform empirical distribution. They were shown to achieve the rate-distortion bound for single description coding of general finite alphabet sources, when used with the strong typicality encoding rule. 


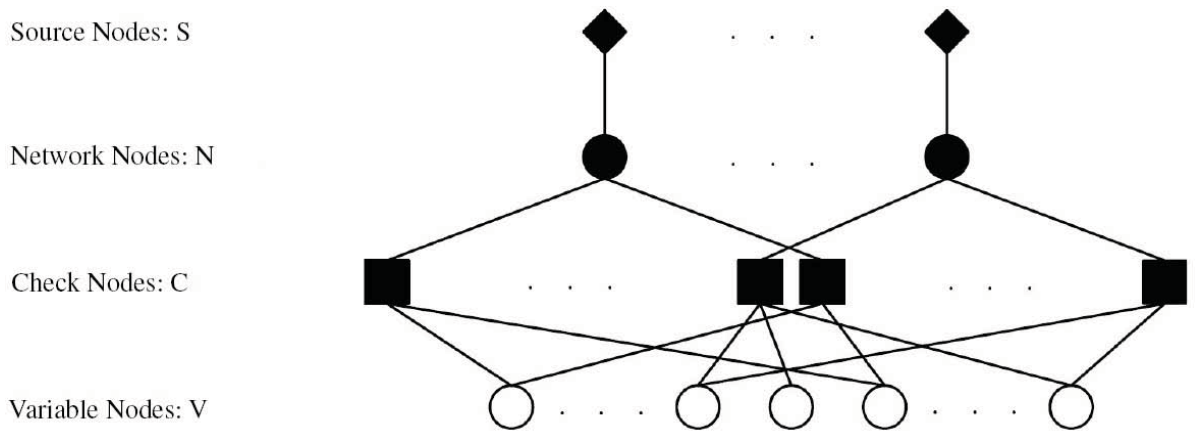

Fig. 1. Factor graph of a multilevel LDGM code.

We mention that a three-stage successive coding scheme based on LDGM codes for the multiple description problem was proposed in [20]. However, the purpose of the three component encoders in [20] differs from our case. Precisely, in [20], the first stage generates a common part of the two descriptions, while the second and third stages produce the remaining part of description 1 , respectively description 2 .

Next we briefly review the design of multilevel LDGM codes under a uniform framework and clarify the specifics for each encoding stage. Notice that the design requirements for each codebook specify the output alphabet $\mathcal{Z}$ of the codewords, the size $2^{n R}$ of the codebook and $n$ probability distributions $p_{1}(\cdot), \cdots, p_{n}(\cdot)$ over $\mathcal{Z}$. The requirement for the codebook is to be randomly generated according to $\prod_{l=1}^{n} p_{l}(\cdot)$. Precisely, we have $p_{l}(\cdot)=p_{X_{1}}(\cdot)$ for $\mathcal{E}_{1}, p_{l}(\cdot)=p_{X_{2}}(\cdot)$ for $\mathcal{E}_{2}$, and $p_{l}(\cdot)=p_{X_{0} \mid X_{1} X_{2}}\left(\cdot \mid x_{1, k_{1}^{*}}(l), x_{2, k_{2}^{*}}(l)\right)$ for $\mathcal{E}_{3}$, $1 \leq l \leq n$.

To approximately satisfy the above requirement, we select an integer $\omega>0$ and $n$ mappings $\phi_{l}:\{0,1\}^{\omega} \rightarrow \mathcal{Z}$ such that $\left|\phi_{l}^{-1}(z)\right| \approx 2^{\omega} p_{l}(z)$ for all $1 \leq l \leq n$ and $z \in \mathcal{Z}$. Based on these mappings, the function $\Phi:\{0,1\}^{n \omega} \rightarrow \mathcal{Z}^{n}$ is defined as follows: the $l$-th symbol of $\Phi\left(c^{n \omega}\right)$ equals $\phi_{l}(c(l), c(n+$ $l), \cdots, c(n(\omega-1)+l))$ for all $c^{n \omega} \in\{0,1\}^{n \omega}$. Further, a low-density generator matrix $G$ of dimension $n \omega \times m$, over the binary field $G F(2)$ is chosen, where $m=n R$. Then the codebook generated by the multilevel LDGM code is defined as

$$
\mathcal{C}=\left\{z^{n} \in \mathcal{Z}^{n} \mid z^{n}=\Phi\left(G v^{m}\right), v^{m} \in\{0,1\}^{m}\right\},
$$

where the matrix multiplication is performed over $G F(2)$.

The multilevel LDGM code is associated with a factor graph as illustrated in Figure 1. The graph consists of $n$ source nodes $\left\{S_{1}, \cdots, S_{n}\right\}$, corresponding to the sequence input to the encoder, $m$ variable nodes $\left\{V_{1}, \cdots, V_{m}\right\}, n \omega$ check nodes $\left\{C_{1}, \cdots, C_{n \omega}\right\}$, and $n$ network nodes $\left\{N_{1}, \cdots, N_{n}\right\}$. Each variable node $V_{k}$ is associated with information bit $v(k)$ and is connected by an edge to every check node $C_{q}$ such that $G(q, k)=1$. Every check node $C_{q}$ is assigned a bit value $c(q)$ computed as the modulo 2 summation of the bit values at adjacent variables nodes. Finally, each network node $N_{l}$ is connected by an edge to check nodes $C_{l}, C_{l+n}, \cdots, C_{l+(\omega-1) n}$, and to the source node $S_{l} . N_{l}$ is associated with the $l$-th symbol $z(l)$ of the codeword, computed by applying the mapping $\phi_{l}(\cdot)$ to the bit values at the adjacent check nodes. The construction of the mapping $\phi_{l}(\cdot)$ ensures that the marginal distribution of symbol $z(l)$ approximates $p_{l}(\cdot)$.

Notice that encoder $\mathcal{E}_{3}$ needs multiple conditional codebooks. However, by choosing a common value of the integer $\omega$ and a common low-density generator matrix $G$ for all these codes, the associated factor graphs become identical. What differs from one codebook to another are only the functions $\phi_{l}(\cdot)$. Since the number of different mappings $\phi_{l}(\cdot)$ is small, the storage space needed at $\mathcal{E}_{3}$ is comparable with that for a single LDGM code.

Each encoder is associated with a pair of random variables $Y$ and $Z$ jointly distributed over the alphabets $\mathcal{Y}$ and $\mathcal{Z}$, respectively. The encoder requirement is, given the input sequence $y^{n}$, to select a codeword $z^{n}$ jointly strongly typical with $y^{n}$. The input sequence $y^{n}$ is the source sequence $x^{n}$ combined with the codeword(s) selected by previous encoder(s), if any. Thus, the alphabet $\mathcal{Y}$ is the cartesian product of $\mathcal{X}$ and the codeword alphabets of previous encoder(s), if any. Precisely, for $\mathcal{E}_{1}$ we have $(Y, Z)=\left(X, X_{1}\right)$ and $y^{n}=x^{n}$. For $\mathcal{E}_{2}$ we have $Y=\left(X, X_{1}\right), Z=X_{2}$, and $y(l)=$ $\left(x(l), x_{1, k_{1}^{*}}(l)\right), 1 \leq l \leq n$. Finally, for $\mathcal{E}_{3}$, we have $Y=$ $\left(X, X_{1}, X_{2}\right), Z=X_{0}$, and $y(l)=\left(x(l), x_{1, k_{1}^{*}}(l), x_{2, k_{2}^{*}}(l)\right)$, $1 \leq l \leq n$.

As in prior work on LDGM-based coding [19]-[22], we use a message passing algorithm over the associated factor graph as a heuristic to solve the encoder problem. Our algorithm of choice is belief propagation with decimation. It proceeds in a series of rounds. Each round consists of a message passing phase where messages are transmitted between every adjacent nodes in a series of iterations, followed by a decimation phase where some variable nodes are fixed and removed from the factor graph. The algorithm stops when all variable nodes are fixed.

At each message passing iteration, every node $A$ passes a message to each adjacent non-source node $B$. If $A$ is not a source node then the message consists of two components: $M_{A \rightarrow B}(0)$ and $M_{A \rightarrow B}(1)$. If $A$ is a source node, the message consists of $|\mathcal{Z}|$ components: $M_{A \rightarrow B}(z), z \in \mathcal{Z}$.

During the first iteration in the first round, only the source nodes and check nodes pass messages, the messages sent by check nodes being $(0.5,0.5)$. After that, at each iteration the schedule of message transmission is: 1) from network nodes and variable nodes to check nodes; 2) from source nodes and check nodes to their adjacent nodes. Every non-source node computes the message to pass along an edge using 


$$
\begin{aligned}
& M_{S_{l} \rightarrow N_{l}}(z)=\exp (-\lambda(y(l), z)), \\
& \text { for all } z \in \mathcal{Z}, 1 \leq l \leq n \text {, }
\end{aligned}
$$

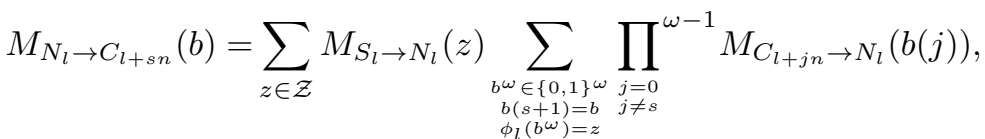

$$
\begin{aligned}
& M_{C_{l+s n} \rightarrow N_{l}}(b)=\frac{1}{2}+\frac{(-1)^{b}}{2} \prod_{k \in \mathcal{B}_{v}(l+s n)}\left(M_{V_{k} \rightarrow C_{l+s n}}(0)-M_{V_{k} \rightarrow C_{l+s n}}(1)\right), \\
& \text { for all } b \in\{0,1\}, 1 \leq l \leq n, 0 \leq s \leq \omega-1 \text {, } \\
& M_{C_{l+s n} \rightarrow V_{k}}(b)=\frac{1}{2}+\frac{(-1)^{b}}{2}\left(M_{N_{l} \rightarrow C_{l+s n}}(0)-M_{N_{l} \rightarrow C_{l+s n}}(1)\right) \prod_{i \in \mathcal{B}_{v}(l+s n) \backslash\{k\}}\left(M_{V_{i} \rightarrow C_{l+s n}}(0)-M_{V_{i} \rightarrow C_{l+s n}}(1)\right) \text {, } \\
& M_{V_{k} \rightarrow C_{l+s n}}(b)=\prod_{q \in \mathcal{A}_{c}(k) \backslash\{l+s n\}} M_{C_{q} \rightarrow V_{k}}(b), \\
& \text { for all } b \in\{0,1\}, k \in \mathcal{B}_{v}(l+s n), 1 \leq l \leq n, 0 \leq s \leq \omega-1 \text {. }
\end{aligned}
$$

Fig. 2. Message passing equations. After applying these equations, the components of each message are normalized to sum up to 1.

the messages received along its other adjacent edges at the previous iteration. The equations to calculate the messages are presented in Figure 2 . We have denoted by $\mathcal{A}_{c}(k)$ the set of indices $q$ such that $C_{q}$ is adjacent to node $V_{k}$, and by $\mathcal{B}_{v}(q)$ the set of of indices $k$ such that $V_{k}$ is adjacent to $C_{q}$. The quantities $\lambda(y, z) \geq 0, y \in \mathcal{Y}, z \in \mathcal{Z}$, used in defining the source messages, are parameters tuned during code design phase based on simulations. As a guideline for selecting these parameters we use the intuition that $\lambda(y, z)$ should be (roughly speaking) inverse proportional to $p_{Z \mid Y}(z \mid y)$.

The message passing phase ends when all the messages $M_{V_{k} \rightarrow C_{q}}(0)$ converge or a maximum number of iterations, typically 100 , is reached. At the decimation phase, the marginal distributions at variable nodes are computed and the variable nodes whose bias is greater than a threshold $\eta>0$ are fixed. If no such variable node exists then the one with highest bias is fixed. After that the fixed variables are removed from the graph. The check node whose all adjacent variable nodes are fixed will send to the adjacent network node the following message: $M(0)=\frac{1}{\gamma}((1-c) \exp (\delta)+c \exp (-\delta))$ and $M(1)=\frac{1}{\gamma}(c \exp (\delta)+(1-c) \exp (-\delta))$, where $c$ equals to the modulo-2 summation of the values of all adjacent variable nodes. Finally, after all variable nodes are fixed, the output codeword is determined on network nodes by mapping the connected check nodes values.

Notice that the design of the proposed scheme does not depend on the distortion measure, but only on the joint distribution $p_{X X_{1} X_{2} X_{0}}$. Therefore, although we have tested this scheme only for Hamming distortion measure, we hypothesize that it is applicable to any distortion function. To support this claim it is worth mentioning that the simulation results in [19] show very good performance of multilevel LDGM codes in the case of single description source coding with a non-Hamming distortion measure.
TABLE I

VALUES OF PARAMETER $\delta$ USED IN SIMULATIONS.

\begin{tabular}{|c|c|c|c|}
\hline$P_{i}$ & $\mathcal{E}_{1}$ & $\mathcal{E}_{2}$ & $\mathcal{E}_{3}$ \\
\hline$P_{1}$ & 0.7 & 1.6 & 1.6 \\
\hline$P_{2}$ & 0.7 & 1.6 & 1.6 \\
\hline$P_{3}$ & 0.7 & 4 & 4 \\
\hline
\end{tabular}

TABLE II

VALUES OF PARAMETERS $\lambda\left(\left(x, x_{1}\right), x_{2}\right)$ AT ENCODER $\mathcal{E}_{2}$.

\begin{tabular}{|c|c|c|c|}
\hline$x, x_{1}, x_{2}$ & $P_{1}$ & $P_{2}$ & $P_{3}$ \\
\hline \hline $0,0,0$ & 0 & 0.6 & 0 \\
\hline $0,0,1$ & 0 & 0 & 0 \\
\hline $0,1,0$ & 0 & 0 & 0 \\
\hline $0,1,1$ & 2.0 & 2.2 & 8 \\
\hline $1,0,0$ & 2.8 & 2.8 & 8 \\
\hline $1,0,1$ & 0 & 0 & 0 \\
\hline $1,1,0$ & 2.8 & 2.8 & 9 \\
\hline $1,1,1$ & 0 & 0 & 0 \\
\hline
\end{tabular}

\section{EXPERIMENTAL RESUlts}

We have tested the proposed robust multiresolution coding scheme for the binary uniform source with Hamming distortion measure, targeting three distortion triples $\left(D_{0}, D_{1}, D_{2}^{*}\left(D_{1}, D_{0}\right)\right): \quad P_{1}=(0.1,0.3,0.3), \quad P_{2}=$ $(0.05,0.3,0.25)$ and $P_{3}=(0,0.3,0.2)$. In all three cases, $R_{1}=R\left(D_{1}\right)$ and $R_{2}=R\left(D_{0}\right)-R\left(D_{1}\right)$ hold. The degree distributions of the LDGM codes used in our tests are taken from the website (http://lthcwww.epfl.ch.research/ldpcopt) or obtained by implementing the algorithm in [23]. We use damping as in [20], [22] in our message passing algorithm, if the messages do not converge after 30 iterations.

The length of the input sequences in our tests is $n=$ 10,000 . We use $\eta=0.9$ and $\omega=4$. The values of parameter 
TABLE III

VALUES OF PARAMETERS $\lambda\left(\left(x, x_{1}, x_{2}\right), x_{0}\right)$ AT ENCODER $\mathcal{E}_{3}$.

\begin{tabular}{|c|c|c|c|}
\hline$x, x_{1}, x_{2}, x_{0}$ & $P_{1}$ & $P_{2}$ & $P_{3}$ \\
\hline \hline $0,0,1,0$ & 0 & 0 & 0 \\
\hline $0,0,1,1$ & 2.8 & 2.8 & 10 \\
\hline $1,0,1,0$ & 1.8 & 2.6 & 10 \\
\hline $1,0,1,1$ & 0 & 0 & 0 \\
\hline
\end{tabular}

TABLE IV

Test Results: $\left(D_{0}, D_{1}, D_{2}^{*}\left(D_{1}, D_{0}\right)\right)$ IS A target Distortion triple; $R_{1}$ AND $R_{2}$ ARE THE RATES OF THE BASE, RESPECTIVELY, REFINEMENT LAYER; $\hat{D}_{0}, \hat{D}_{1}$, AND $\hat{D}_{2}$ ARE THE EMPIRICAL DISTORTIONS.

\begin{tabular}{|c|c|c|c|c|}
\hline$\left(R_{1}, R_{2}\right)$ & $\left(D_{0}, D_{1}, D_{2}^{*}\left(D_{1}, D_{0}\right)\right)$ & $\hat{D}_{0}$ & $\hat{D}_{1}$ & $\hat{D}_{2}$ \\
\hline \hline$(0.1187,0.4122)$ & $(0.10,0.30,0.30)$ & 0.109 & 0.308 & 0.304 \\
\hline$(0.1187,0.5955)$ & $(0.05,0.30,0.25)$ & 0.066 & 0.308 & 0.258 \\
\hline$(0.1187,0.8813)$ & $(0.00,0.30,0.20)$ & 0.012 & 0.309 & 0.206 \\
\hline
\end{tabular}

$\delta$ are listed in Table I. To define the source messages for $\mathcal{E}_{1}$, we use $\lambda(0,0)=\lambda(1,1)=0$ and $\lambda(0,1)=\lambda(1,0)=0.7$. The values of $\lambda(y, z)$ for encoders $\mathcal{E}_{2}$, and $\mathcal{E}_{3}$ are presented in Tables II and III, respectively. It is worth mentioning that for all three cases $P_{1}, P_{2}, P_{3}$, variable $X_{0}$ is a deterministic function of $X_{1}$ and $X_{2}$ when $\left(X_{1}, X_{2}\right) \neq(0,1)$. Thus, at encoder $\mathcal{E}_{3}$, for $l$ such that $\left(x_{1, k_{1}^{*}}(l), x_{2, k_{2}^{*}}(l)\right) \neq(0,1)$, the network node $N_{l}$ always sends the uniform message $(0.5,0.5)$, irrespective of the message received from $S_{l}$. Therefore, we set $\lambda\left(\left(x, x_{1}, x_{2}\right), x_{0}\right)=0$ for all binary quadruples $\left(x, x_{1}, x_{2}, x_{0}\right)$ with $\left(x_{1}, x_{2}\right) \neq(0,1)$.

Table IV presents the experimental results. The first column contains the rates $R_{1}, R_{2}$ of the base, respectively, refinement layer. The second column contains the target distortion triple $\left(D_{0}, D_{1}, D_{2}\right)$. The remaining three columns present the empirical values of the three distortions, respectively, averaged over 100 runs. As observed from Table IV, the distortions are very close to the theoretical limits.

\section{CONCLUSION}

This work derives an explicit expression of the minimum distortion achievable by the refinement layer of a multiresolution code for arbitrary finite alphabet sources with Hamming distortion measure. A practical robust multiresolution coding scheme based on LDGM codes is proposed, which shows promising performance.

\section{APPENDIX A}

Proof of LEMMA 2

For any $X_{2}$ such that $I\left(X ; X_{1}, X_{2} \mid X_{0}\right)=0$ and $I\left(X_{1} ; X_{2}\right)=0$, define $\tilde{X}_{2}=X_{2}$ if $X_{2} \in \mathcal{X}_{0}^{+}$and $\tilde{X}_{2}=0$ if $X_{2} \notin \mathcal{X}_{0}^{+}$. It is clear that $I\left(X ; X_{1}, \tilde{X}_{2} \mid X_{0}\right)=0$ and

$$
\begin{aligned}
& I\left(X_{1} ; \tilde{X}_{2}\right)=0 . \text { Note that for } x_{0} \in \mathcal{X}_{0}^{+} \\
& \qquad \begin{aligned}
p_{X \mid X_{0}}\left(0 \mid x_{0}\right) & \geq \min \left(1-D_{0}, \lambda_{0}\right) \\
& \geq \min \left(1-\left.D(R)\right|_{R=0}, \lambda_{0}\right) \\
& =\min \left(p_{X}(0), \lambda_{0}\right) \\
& =\lambda_{0} \\
& \geq \max _{x \notin \mathcal{X}_{0}^{+}} p_{X \mid X_{0}}\left(x \mid x_{0}\right),
\end{aligned}
\end{aligned}
$$

where the first equality follows from F1), and the second equality follows from the fact that $\lambda_{0} \leq \lambda_{1} \leq p_{X}(1) \leq p_{X}(0)$ (see F3)). Therefore, we have

$$
\begin{aligned}
\mathbb{P}\left(X=X_{2}\right)= & \sum_{x_{0} \in \mathcal{X}_{0}^{+}} \sum_{x_{2} \in \mathcal{X}} p_{X X_{0} X_{2}}\left(x_{2}, x_{0}, x_{2}\right) \\
= & \sum_{x_{0} \in \mathcal{X}_{0}^{+}} \sum_{x_{2} \in \mathcal{X}} p_{X \mid X_{0}}\left(x_{2} \mid x_{0}\right) p_{X_{0} X_{2}}\left(x_{0}, x_{2}\right) \\
= & \sum_{x_{0} \in \mathcal{X}_{0}^{+}}\left(\sum_{x_{2} \in \mathcal{X}_{0}^{+}} p_{X \mid X_{0}}\left(x_{2} \mid x_{0}\right) p_{X_{0} X_{2}}\left(x_{0}, x_{2}\right)\right. \\
& \left.+\sum_{x_{2} \notin \mathcal{X}_{0}^{+}} p_{X \mid X_{0}}\left(x_{2} \mid x_{0}\right) p_{X_{0} X_{2}}\left(x_{0}, x_{2}\right)\right) \\
\leq & \sum_{x_{0} \in \mathcal{X}_{0}^{+}}\left(\sum_{x_{2} \in \mathcal{X}_{0}^{+}} p_{X \mid X_{0}}\left(x_{2} \mid x_{0}\right) p_{X_{0} X_{2}}\left(x_{0}, x_{2}\right)\right. \\
& \left.+p_{X \mid X_{0}}\left(0 \mid x_{0}\right) \sum_{x_{2} \notin \mathcal{X}_{0}^{+}} p_{X_{0} X_{2}}\left(x_{0}, x_{2}\right)\right) \\
= & \sum_{x_{0} \in \mathcal{X}_{0}^{+}}\left(p _ { X | X _ { 0 } } ( 0 | x _ { 0 } ) \left(p_{X_{0} X_{2}}\left(x_{0}, 0\right)\right.\right. \\
& \left.+\sum_{x_{2} \notin \mathcal{X}_{0}^{+}} p_{X_{0} X_{2}}\left(x_{0}, x_{2}\right)\right) \\
& \left.+\sum_{x_{2} \in \mathcal{X}_{0}^{+}, x_{2} \neq 0} p_{X \mid X_{0}}\left(x_{2} \mid x_{0}\right) p_{X_{0} X_{2}}\left(x_{0}, x_{2}\right)\right) \\
= & \sum_{x_{0} \in \mathcal{X}_{0}^{+}} \sum_{\tilde{x}_{2} \in \mathcal{X}_{0}^{+}} p_{X \mid X_{0}}\left(\tilde{x}_{2} \mid x_{0}\right) p_{X_{0} \tilde{X}_{2}}\left(x_{0}, \tilde{x}_{2}\right) \\
= & \mathbb{P}\left(X=\tilde{X}_{2}\right) .
\end{aligned}
$$

\section{APPENDIX B}

PROOF OF LEMMA 3

Note that

$$
\begin{aligned}
& \mathbb{P}\left(X=X_{2}\right) \\
& =\sum_{x_{0} \in \mathcal{X}_{0}^{+}} \sum_{x_{2} \in \mathcal{X}_{0}^{+}} p_{X X_{0} X_{2}}\left(x_{2}, x_{0}, x_{2}\right) \\
& =\sum_{x_{0} \in \mathcal{X}_{0}^{+}} \sum_{x_{2} \in \mathcal{X}_{0}^{+}} p_{X \mid X_{0}}\left(x_{2} \mid x_{0}\right) p_{X_{0} X_{2}}\left(x_{0}, x_{2}\right) \\
& =\sum_{x_{0} \in \mathcal{X}_{0}^{+}}\left(p_{X \mid X_{0}}\left(x_{0} \mid x_{0}\right) p_{X_{0} X_{2}}\left(x_{0}, x_{0}\right)\right. \\
& \left.\quad+\sum_{x_{2} \in \mathcal{X}_{0}^{+}, x_{2} \neq x_{0}} p_{X \mid X_{0}}\left(x_{2} \mid x_{0}\right) p_{X_{0} X_{2}}\left(x_{0}, x_{2}\right)\right) \\
& =\sum_{x_{0} \in \mathcal{X}_{0}^{+}}\left(\left(1-D_{0}\right) p_{X_{0} X_{2}}\left(x_{0}, x_{0}\right)\right.
\end{aligned}
$$




$$
\begin{aligned}
& \left.+\sum_{x_{2} \in \mathcal{X}_{0}^{+}, x_{2} \neq x_{0}} \lambda_{0} p_{X_{0} X_{2}}\left(x_{0}, x_{2}\right)\right) \\
& =\left(1-D_{0}\right) \mathbb{P}\left(X_{0}=X_{2}\right)+\lambda_{0} \mathbb{P}\left(X_{0} \neq X_{2}\right),
\end{aligned}
$$

where (17) is due to (3). Therefore, we have

$$
\begin{aligned}
\mathbb{P}\left(X \neq X_{2}\right) & =1-\mathbb{P}\left(X=X_{2}\right) \\
& =1-\left(1-D_{0}\right) \mathbb{P}\left(X_{0}=X_{2}\right)-\lambda_{0} \mathbb{P}\left(X_{0} \neq X_{2}\right) \\
& =D_{0}+\left(1-D_{0}-\lambda_{0}\right) \mathbb{P}\left(X_{0} \neq X_{2}\right) .
\end{aligned}
$$

APPENDIX C

PRoOF OF LEMMA 5

It is easy to see that

$$
\begin{aligned}
& \bar{D}_{2}\left(D_{0}, D_{1}\right) \\
& =\min _{p_{X_{0} X_{1} X_{2}}} 1-\sum_{x_{0} \in \mathcal{X}_{0}^{+}} \sum_{x_{1} \in \mathcal{X}_{1}^{+}} p_{X_{0} X_{1} X_{2}}\left(x_{0}, x_{1}, x_{0}\right)
\end{aligned}
$$

subject to the constraints

$$
\begin{gathered}
-p_{X_{0} X_{1} X_{2}}\left(x_{0}, x_{1}, x_{2}\right) \leq 0, \\
x_{0} \in \mathcal{X}_{0}^{+}, x_{1} \in \mathcal{X}_{1}^{+}, x_{2} \in \mathcal{X}_{0}^{+}, \\
\sum_{x_{2} \in \mathcal{X}_{0}^{+}} p_{X_{0} X_{1} X_{2}}\left(x_{0}, x_{1}, x_{2}\right)=p_{X_{0} X_{1}}\left(x_{0}, x_{1}\right), \\
x_{0} \in \mathcal{X}_{0}^{+}, x_{1} \in \mathcal{X}_{1}^{+}, \\
p_{X_{1}}\left(x_{1}\right) \sum_{x_{0} \in \mathcal{X}_{0}^{+}} p_{X_{0} X_{1} X_{2}}\left(x_{0}, 0, x_{2}\right) \\
-p_{X_{1}}(0) \sum_{x_{0} \in \mathcal{X}_{0}^{+}} p_{X_{0} X_{1} X_{2}}\left(x_{0}, x_{1}, x_{2}\right)=0, \\
x_{1} \in \mathcal{X}_{1}^{+} \backslash\{0\}, x_{2}=\mathcal{X}_{0}^{+} \backslash\{0\},
\end{gathered}
$$

where (19) and (20) are due to the fact that $p_{X_{0} X_{1} X_{2}}$ is a probability distribution and that $p_{X_{0} X_{1}}$ is fixed while (21) is due to the independence of $X_{1}$ and $X_{2}$.

Since (18) is a linear programming problem, the Karush-Kuhn-Tucker conditions are sufficient for global optimality. Now introduce Lagrangian multipliers $\mu=$

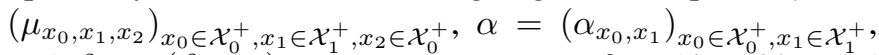
and $\beta=\left(\beta_{x_{1}, x_{2}}\right)_{x_{1} \in \mathcal{X}_{1}^{+} \backslash\{0\}, x_{2} \in \mathcal{X}_{0}^{+} \backslash\{0\}}$ for (19), (20), and (21), respectively. Define

$$
\begin{aligned}
G & \left(p_{X_{0} X_{1} X_{2}}, \mu, \alpha, \beta\right) \\
= & 1-\sum_{x_{0} \in \mathcal{X}_{0}^{+}} \sum_{x_{1} \in \mathcal{X}_{1}^{+}} p_{X_{0} X_{1} X_{2}}\left(x_{0}, x_{1}, x_{0}\right) \\
& -\sum_{x_{0} \in \mathcal{X}_{0}^{+}} \sum_{x_{1} \in \mathcal{X}_{1}^{+}} \sum_{x_{0} \in \mathcal{X}_{0}^{+}} \mu_{x_{0}, x_{1}, x_{2}} p_{X_{0} X_{1} X_{2}}\left(x_{0}, x_{1}, x_{2}\right) \\
& +\sum_{x_{0} \in \mathcal{X}_{0}^{+}} \sum_{x_{1} \in \mathcal{X}_{1}^{+}} \alpha_{x_{0}, x_{1}} \sum_{x_{2} \in \mathcal{X}_{0}^{+}} p_{X_{0} X_{1} X_{2}}\left(x_{0}, x_{1}, x_{2}\right) \\
+ & \sum_{x_{1} \in \mathcal{X}_{1}^{+} \backslash\{0\}} \sum_{x_{2} \in \mathcal{X}_{0}^{+} \backslash\{0\}} \beta_{x_{1}, x_{2}} \sum_{x_{0} \in \mathcal{X}_{0}^{+}}\left(p_{X_{1}}\left(x_{1}\right)\right. \\
& \left.\times p_{X_{0} X_{1} X_{2}}\left(x_{0}, 0, x_{2}\right)-p_{X_{1}}(0) p_{X_{0} X_{1} X_{2}}\left(x_{0}, x_{1}, x_{2}\right)\right) .
\end{aligned}
$$

The Karush-Kuhn-Tucker conditions are given by

$\frac{\partial G\left(p_{X_{0} X_{1} X_{2}}, \mu, \alpha, \beta\right)}{\partial p_{X_{0} X_{1} X_{2}}\left(x_{0}, x_{1}, x_{2}\right)}=0, \quad x_{0} \in \mathcal{X}_{0}^{+}, x_{1} \in \mathcal{X}_{1}^{+}, x_{2} \in \mathcal{X}_{0}^{+}$,

$-p_{X_{0} X_{1} X_{2}}\left(x_{0}, x_{1}, x_{2}\right) \leq 0, \quad x_{0} \in \mathcal{X}_{0}^{+}, x_{1} \in \mathcal{X}_{1}^{+}, x_{2} \in \mathcal{X}_{0}^{+}$,

$\sum_{x_{2} \in \mathcal{X}_{0}^{+}} p_{X_{0} X_{1} X_{2}}\left(x_{0}, x_{1}, x_{2}\right)=p_{X_{0} X_{1}}\left(x_{0}, x_{1}\right)$,

$$
\begin{gathered}
x_{0} \in \mathcal{X}_{0}^{+}, x_{1} \in \mathcal{X}_{1}^{+}, \\
p_{X_{1}}\left(x_{1}\right) \sum_{x_{0} \in \mathcal{X}_{0}^{+}} p_{X_{0} X_{1} X_{2}}\left(x_{0}, 0, x_{2}\right) \\
-p_{X_{1}}(0) \sum_{x_{0} \in \mathcal{X}_{0}^{+}} p_{X_{0} X_{1} X_{2}}\left(x_{0}, x_{1}, x_{2}\right)=0, \\
x_{1} \in \mathcal{X}_{1}^{+} \backslash\{0\}, x_{2}=\mathcal{X}_{0}^{+} \backslash\{0\},
\end{gathered}
$$

$\mu_{x_{0}, x_{1}, x_{2}} \geq 0, \quad x_{0} \in \mathcal{X}_{0}^{+}, x_{1} \in \mathcal{X}_{1}^{+}, x_{2} \in \mathcal{X}_{0}^{+}$,

$\mu_{x_{0}, x_{1}, x_{2}} p_{X_{0} X_{1} X_{2}}\left(x_{0}, x_{1}, x_{2}\right)=0$,

$$
x_{0} \in \mathcal{X}_{0}^{+}, x_{1} \in \mathcal{X}_{1}^{+}, x_{2} \in \mathcal{X}_{0}^{+},
$$

where (22) is equivalent to

$$
\begin{aligned}
& d_{H}\left(x_{0}, x_{2}\right)-1-\mu_{x_{0}, x_{1}, x_{2}}+\alpha_{x_{0}, x_{1}} \\
& +\sum_{x_{1}^{\prime} \in \mathcal{X}_{1}^{+} \backslash\{0\}} \beta_{x_{1}^{\prime}, x_{2}} p_{X_{1}}\left(x_{1}^{\prime}\right)=0,
\end{aligned}
$$

$$
\begin{array}{r}
x_{0} \in \mathcal{X}_{0}^{+}, x_{1}=0, x_{2} \in \mathcal{X}_{1}^{+} \backslash\{0\}, \\
d_{H}\left(x_{0}, x_{2}\right)-1-\mu_{x_{0}, x_{1}, x_{2}}+\alpha_{x_{0}, x_{1}}-\beta_{x_{1}, x_{2}} p_{X_{1}}(0)=0, \\
x_{0} \in \mathcal{X}_{0}^{+}, x_{1} \in \mathcal{X}_{1}^{+} \backslash\{0\}, x_{2} \in \mathcal{X}_{0}^{+} \backslash\{0\}, \\
d_{H}\left(x_{0}, x_{2}\right)-1-\mu_{x_{0}, x_{1}, x_{2}}+\alpha_{x_{0}, x_{1}}=0, \\
x_{0} \in \mathcal{X}_{0}^{+}, x_{1} \in \mathcal{X}_{1}^{+}, x_{2}=0 .
\end{array}
$$

Let

$$
\begin{aligned}
& p_{X_{0} X_{1} X_{2}^{*}}\left(x_{0}, x_{1}, x_{2}\right) \\
& = \begin{cases}p_{X_{0} X_{1}}\left(x_{1}, x_{1}\right)-p_{X_{0} X_{1}}\left(0, x_{1}\right), & x_{0}=x_{1} \neq x_{2}=0 \\
p_{X_{0} X_{1}}\left(x_{0}, x_{1}\right), & x_{0}=x_{2} \neq x_{1} \\
p_{X_{0} X_{1}}\left(0, x_{1}\right), & x_{0}=x_{1}=x_{2} \\
0, & \text { otherwise }\end{cases}
\end{aligned}
$$

for $x_{0} \in \mathcal{X}_{0}^{+}, x_{1} \in \mathcal{X}_{1}^{+}$, and $x_{2} \in \mathcal{X}_{0}^{+}$. Let

$\mu_{x_{0}, x_{1}, x_{2}}^{*}=0, \quad x_{0}=x_{2} \in \mathcal{X}_{0}^{+}, x_{1} \in \mathcal{X}_{1}^{+}$,

$\mu_{x_{0}, x_{1}, x_{2}}^{*}=0, \quad x_{0}=x_{1} \in \mathcal{X}_{1}^{+} \backslash\{0\}, x_{2}=0$,

$\mu_{x_{0}, x_{1}, x_{2}}^{*}=\alpha_{x_{0}, x_{1}}^{*}$,

$x_{0} \neq x_{1}, x_{2}=0, x_{0} \in \mathcal{X}_{0}^{+} \backslash\{0\}, x_{1} \in \mathcal{X}_{1}^{+}$,

$\mu_{x_{0}, x_{1}, x_{2}}^{*}=\alpha_{x_{0}, x_{1}}^{*}+\sum_{x_{1}^{\prime} \in \mathcal{X}_{1}^{+} \backslash\{0\}} \beta_{x_{1}^{\prime}, x_{2}}^{*} p_{X_{1}}\left(x_{1}^{\prime}\right)$,

$x_{0} \neq x_{2}, x_{1}=0, x_{0} \in \mathcal{X}_{0}^{+}, x_{2} \in \mathcal{X}_{0}^{+} \backslash\{0\}$,

$\mu_{x_{0}, x_{1}, x_{2}}^{*}=\alpha_{x_{0}, x_{1}}^{*}-\beta_{x_{1}, x_{2}}^{*} p_{X_{1}}(0)$,

$x_{0} \neq x_{2}, x_{0} \in \mathcal{X}_{0}^{+}, x_{1} \in \mathcal{X}_{1}^{+} \backslash\{0\}, x_{2} \in \mathcal{X}_{0}^{+} \backslash\{0\}$,

$\alpha_{x_{0}, x_{1}}^{*}=0, \quad x_{0}=x_{1} \in \mathcal{X}_{1}^{+} \backslash\{0\}$,

$\alpha_{x_{0}, x_{1}}^{*}=1, \quad x_{0}=0, x_{1} \in \mathcal{X}_{1}^{+}$,

$\alpha_{x_{0}, x_{1}}^{*}=1, \quad x_{0} \neq x_{1}, x_{0} \in \mathcal{X}_{0}^{+} \backslash\{0\}, x_{1} \in \mathcal{X}_{1}^{+} \backslash\{0\}$, 


$$
\begin{aligned}
& \alpha_{x_{0}, x_{1}}^{*}=\frac{1-\sum_{x_{1}^{\prime} \neq x_{0}, x_{1}^{\prime} \in \mathcal{X}_{1}^{+} \backslash\{0\}} p_{X_{1}}\left(x_{1}^{\prime}\right)}{p_{X_{1}}(0)}, \\
& x_{0} \in \mathcal{X}_{0}^{+} \backslash\{0\}, x_{1}=0, \\
& \beta_{x_{1}, x_{2}}^{*}=\frac{\alpha_{x_{2}, x_{1}}^{*}-1}{p_{X_{1}}(0)}, \quad x_{1} \in \mathcal{X}_{1}^{+} \backslash\{0\}, x_{2} \in \mathcal{X}_{0}^{+} \backslash\{0\} .
\end{aligned}
$$

It can be verified that the Karush-Kuhn-Tucker conditions are satisfied by the constructed $\left(p_{X_{0} X_{1} X_{2}^{*}}, \mu^{*}, \alpha^{*}, \beta^{*}\right)$. Moreover, note that

$$
\begin{aligned}
& 1-\sum_{x_{0} \in \mathcal{X}_{0}^{+}} \sum_{x_{1} \in \mathcal{X}_{1}^{+}} p_{X_{0} X_{1} X_{2}^{*}}\left(x_{0}, x_{1}, x_{0}\right) \\
& =1-\sum_{x_{0} \in \mathcal{X}_{1}^{+}} \sum_{x_{1} \in \mathcal{X}_{1}^{+}} p_{X_{0} X_{1} X_{2}^{*}}\left(x_{0}, x_{1}, x_{0}\right) \\
& -\sum_{x_{0} \in \mathcal{X}_{0}^{+} \backslash \mathcal{X}_{1}^{+}} \sum_{x_{1} \in \mathcal{X}_{1}^{+}} p_{X_{0} X_{1} X_{2}^{*}}\left(x_{0}, x_{1}, x_{0}\right) \\
& =1-\sum_{x_{0} \in \mathcal{X}_{1}^{+}}\left(p_{X_{0} X_{1} X_{2}^{*}}\left(x_{0}, x_{0}, x_{0}\right)\right. \\
& \left.+\sum_{x_{1} \neq x_{0}} p_{X_{0} X_{1} X_{2}^{*}}\left(x_{0}, x_{1}, x_{0}\right)\right) \\
& -\sum_{x_{0} \in \mathcal{X}_{0}^{+} \backslash \mathcal{X}_{1}^{+}} \sum_{x_{1} \in \mathcal{X}_{1}^{+}} p_{X_{0} X_{1} X_{2}^{*}}\left(x_{0}, x_{1}, x_{0}\right) \\
& =1-\sum_{x_{0} \in \mathcal{X}_{1}^{+}}\left(p_{X_{0} X_{1}}\left(0, x_{0}\right)+\sum_{x_{1} \neq x_{0}} p_{X_{0} X_{1}}\left(x_{0}, x_{1}\right)\right) \\
& -\sum_{x_{0} \in \mathcal{X}_{0}^{+} \backslash \mathcal{X}_{1}^{+}} \sum_{x_{1} \in \mathcal{X}_{1}^{+}} p_{X_{0} X_{1}}\left(x_{0}, x_{1}\right) \\
& =1-p_{X_{0}}(0)-\sum_{x_{0} \in \mathcal{X}_{1}^{+}} \sum_{x_{1} \neq x_{0}} p_{X_{0} X_{1}}\left(x_{0}, x_{1}\right) \\
& -\sum_{x_{0} \in \mathcal{X}_{0}^{+} \backslash \mathcal{X}_{1}^{+}} \sum_{x_{1} \in \mathcal{X}_{1}^{+}} p_{X_{0} X_{1}}\left(x_{0}, x_{1}\right) \\
& =1-p_{X_{0}}(0)-\mathbb{P}\left(X_{0} \neq X_{1}\right) \text {. }
\end{aligned}
$$

The proof is complete.

\section{ACKNOWLEDGMENT}

The work of Jun Chen was supported by the Early Research Award from the Province of Ontario and by the Natural Science and Engineering Research Council (NSERC) of Canada under a discovery grant. The work of Sorina Dumitrescu and Ying Zhang was supported by NSERC under a discovery grant. The work of Jia Wang was supported by the NSFC under Grant 60802020 and 973 Program (2010CB731401, 2010CB731406).

\section{REFERENCES}

[1] J. Shapiro, "Embedded image coding using zerotrees of wavelet coefficients," IEEE Trans. Signal Process., vol. 41, pp. 3445-3462, Dec. 1993.

[2] H. Brunk and N. Farvardin, "Fixed-rate successively refinable scalar quantizers," in Proc. DCC'96, Snowbird, Utah, Mar. 1996, pp. 250-259.

[3] A. Said and W. Pearlman, "A new, fast, and efficient image codec based on set partitioning in hierarchical trees," IEEE Trans. Circuits Syst. Video Technol., vol. 6, pp. 243-250, June 1996.

[4] H. Brunk and N. Farvardin, "Embedded entropy-constrained trellis coded quantization," in Proc. International Symp. Inf. Theory, Cambridge, MA, p. 274, Aug. 1998.
[5] H. Jafarkhani and V. Tarokh, "Design of successively refinable trelliscoded quantizers," IEEE Trans. Inf. Theory, vol. 45, pp. 1490-1497, July 1999.

[6] D. Taubman, "High performance scalable image compression with EBCOT," IEEE Trans. Image Process., vol. 9, pp. 1158-1170, July 2000.

[7] S. Dumitrescu and X. Wu, "Algorithms for optimal multi-resolution quantization," J. Algorithms, vol. 50, pp. 1-22, 2004.

[8] M. Effros and D. Dugatkin, "Multiresolution vector quantization," IEEE Trans. Inf. Theory, vol. 50, pp. 3130-3145, Dec. 2004

[9] A. El Gamal and T. Cover, "Achievable rates for multiple descriptions," IEEE Trans. Inf. Theory, vol. 28, pp. 851-857, Nov. 1982.

[10] Z. Zhang and T. Berger, "New results in binary multiple descriptions," IEEE Trans. Inf. Theory, vol. 33, pp. 502-521, July 1987.

[11] R. Venkataramani, G. Kramer, and V. K. Goyal, "Multiple description coding with many channels," IEEE Trans. Inf. Theory, vol. 49, pp. 21062114, Sep. 2003.

[12] B. Rimoldi, "Successive refinement of information: characterization of the achievable rates," IEEE Trans. Inf. Theory, vol. 40, pp. 253-259, Jan. 1994.

[13] J. Wang, J. Chen, L. Zhao, P. Cuff, and H. Permuter, "A random variable substitution lemma with applications to multiple description coding," preprint. [Online]. Available: http://arxiv.org/abs/0909.3135.

[14] R. Ahlswede, "The rate-distortion region for multiple descriptions without excess rate," IEEE Trans. Inf. Theory, vol. 31, pp. 721-726, Nov. 1985.

[15] W. H. R. Equitz and T. M. Cover, "Successive refinement of information," IEEE Trans. Inf. Theory, vol. 37, pp. 269-275, Mar. 1991.

[16] V. Erokhin, "Epsilon-entropy of a discrete random variable," Theory Probability Applications, vol. 3, pp. 97-100, 1958, English translation.

[17] J. T. Pinkston, "An application of rate-distortion theory to a converse to the coding theorem," IEEE Trans. Inf. Theory, vol. IT-15, pp. 66-71, Jan. 1969.

[18] L. Ozarow, "On a source-coding problem with two channels and three receivers," Bell Syst. Tech. J., vol. 59, no. 10, pp. 1909-1921, Dec. 1980.

[19] Z. Sun, M. Shao, J. Chen, K. M. Wong, and X. Wu, "Achieving the ratedistortion bound with low-density generator matrix codes," IEEE Trans. Commun., vol. 58, pp. 1643-1653, June 2010.

[20] Y. Zhang, S. Dumitrescu, J. Chen, and Z. Sun, "LDGM-based multiple description coding with Hamming distortion measure," preprint.

[21] M. Wainwright and E. Maneva, "Lossy source encoding via messagepassing and decimation over generalized codewords of LDGM codes," in Proc. International Symp. Inf. Theory, pp. 1493-1497, Sep. 2005.

[22] T. Filler and J. Friedrich, "Binary quantization using belief propagation with decimation over factor graphs of LDGM codes," in Proc. Allerton Conf. Commun., Control, Comput., Monticello, IL, Sep. 2007.

[23] S.-Y. Chung, G. D. Forney, T. J. Richardson, and R. Urbanke, "On the design of low-desity parity check codes within $0.0045 \mathrm{~dB}$ of the Shannon limit," IEEE Commun. Lett., vol. 5, pp. 58-60, Feb. 2001.

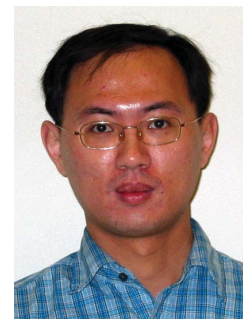

Jun Chen (S'03-M'06) received the B.E. degree with honors in communication engineering from Shanghai Jiao Tong University, Shanghai, China, in 2001 and the M.S. and Ph.D. degrees in electrical and computer engineering from Cornell University, Ithaca, NY, in 2004 and 2006, respectively.

He was a Postdoctoral Research Associate in the Coordinated Science Laboratory at the University of Illinois at Urbana-Champaign, Urbana, IL, from 2005 to 2006, and a Postdoctoral Fellow at the IBM Thomas J. Watson Research Center, Yorktown Heights, NY, from 2006 to 2007. He is currently an Assistant Professor of Electrical and Computer Engineering at McMaster University, Hamilton, ON, Canada. He holds the Barber-Gennum Chair in Information Technology. His research interests include information theory, wireless communications, and signal processing. He received the Josef Raviv Memorial Postdoctoral Fellowship in 2006 and the Early Research Award from the Province of Ontario in 2010. 


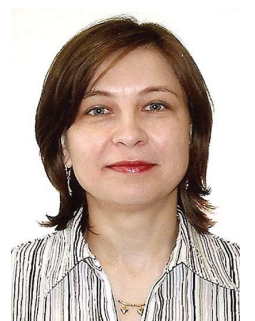

Sorina Dumitrescu (M'04) received the B.Sc. degree in 1990 and the Ph.D. degree in 1997, both in mathematics, from the University of Bucharest, Bucharest, Romania. She is currently an Assistant Professor at the Department of Electrical and Computer Engineering, McMaster University, Hamilton, ON, Canada. Her research interests include multimedia computing and communications, joint sourcechannel coding, signal quantization, steganalysis. Dr. Dumitrescu currently holds an NSERC University Faculty Award.

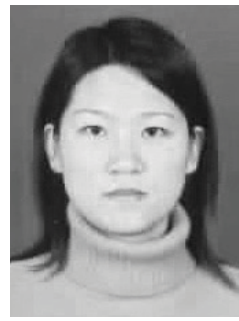

Ying Zhang received the B.Eng degree in $\mathrm{Au}$ tomation from Northeastern University, Shenyang, China in 2004 and the M.Eng degree in Pattern Recognition and Intelligence System from Tianjin University, Tianjin, China in 2007. She is currently pursuing the Ph.D. degree at the Department of Electrical and Computer Engineering, McMaster University, Hamilton, ON, Canada. Her research interests include information theory and multimedia communications.

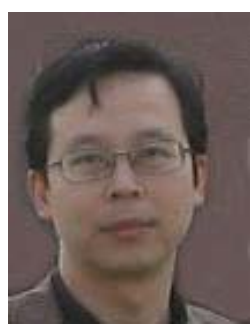

Jia Wang received the B.Sc. degree in electronic engineering, the M.S. degree in pattern recognition and intelligence control, and the Ph.D. degree in electronic engineering from Shanghai Jiao Tong University, China, in 1997, 1999, and 2002, respectively. Since 2002, he has been with the Institute of Image Communication and Information Processing, where he is currently an associate professor. His research interests include multiuser information theory and its application in video coding. 